\section{Tendências de consumo abusivo de álcool nas capitais brasileiras entre os anos de 2006 a 2013: análise das informações do VIGITEL}

\author{
Trends in alcohol abuse in Brazilian state capitals \\ from 2006 to 2013: an analysis of data from the \\ VIGITEL survey
}

\section{Tendencias de consumo abusivo de alcohol en las capitales brasileñas entre los años de 2006 a 2013: análisis de la información del VIGITEL}

\section{Resumo}

Objetivou-se avaliar a tendência do consumo abusivo de álcool no Brasil entre 2006 e 2013 segundo características demográficas, socioeconômicas e regionais. Trata-se da análise de estudos transversais (VIGITEL - Vigilância de Fatores de Risco e Proteção para Doenças Crônicas por Inquérito Telefônico) que avaliaram a população adulta ( $\geq 18$ anos) brasileira residente nas capitais do país. A amostragem foi probabilística, realizada em dois estágios (linha telefônica e morador adulto). Considerou-se consumo abusivo de álcool a ingestão de $\geq 5$ (homem) ou $\geq 4$ (mulher) doses de álcool em uma única ocasião, ao menos uma vez nos 30 dias anteriores à entrevista. A análise de tendência foi obtida usando-se a regressão de Prais-Winsten. A prevalência de consumo abusivo de álcool foi de 15,6\% em 2006 e 16, 4\% em 2013, com tendência estacionária para toda amostra $(p=0,334)$ e para ambos os sexos. A tendência foi crescente entre os idosos e aqueles com 30-39 anos em ambos os sexos e para as mulheres da Região Sudeste. Tendência estacionária foi observada entre os diferentes grupos de escolaridade. Não foram observadas tendências decrescentes no período avaliado.

Consumo de Bebidas Alcoólicas; Bebedeira; Comportamento Perigoso
Tiago N. Munhoz 1

Iná S. Santos 1

Bruno P. Nunes 2

Christian Loret de Mola 2

Inácio Crochemore Mohnsam da Silva 1

Alicia Matijasevich 1,3

doi: $10.1590 / 0102-311 \times 00104516$

\author{
Correspondência \\ T. N. Munhoz \\ Programa de Pós-graduação em Epidemiologia, Universidade \\ Federal de Pelotas. \\ Rua Marechal Deodoro 1160, Pelotas, RS 96020-220, Brasil. \\ tyagomunhoz@hotmail.com \\ 1 Programa de Pós-graduação em Epidemiologia, Universidade \\ Federal de Pelotas, Pelotas, Brasil. \\ 2 Faculdade de Enfermagem, Universidade Federal de Pelotas, \\ Pelotas, Brasil. \\ 3 Faculdade de Medicina, Universidade de São Paulo, São Paulo, \\ Brasil.
}




\section{Introdução}

O álcool é a substância psicoativa mais consumida em todo o mundo 1. Em 2010, a Organização Mundial da Saúde (OMS) estimou que 38,3\% da população mundial com idade $\geq 15$ anos consumiram bebidas alcoólicas no último ano ${ }^{2}$. Considerando todos os países do mundo, a média anual de consumo de álcool por pessoa foi de 6,2 litros 2 , com diferenças entre as regiões do mundo. A fração atribuível à mortalidade por todas as causas relacionadas ao consumo de álcool é de 5,9\% em todo o mundo, sendo maior na Europa (13\%) e menor no Mediterrâneo Oriental (0,9\%). Nas Américas, estima-se que 4,7\% de todas as mortes são atribuídas ao consumo abusivo de álcool.

A permissividade do consumo de álcool na maioria das culturas e sociedades, aliada a seu grande potencial para a dependência física e psicológica, gera um importante prejuízo para a saúde individual e coletiva. Em diferentes países do mundo é observado um aumento no consumo de bebidas alcoólicas entre os homens com idades de 15 a 59 anos 2. Em 2012, a proporção de mortalidade relacionada ao consumo de álcool foi duas vezes maior entre os homens do que entre as mulheres $(7,6 \%$ vs. $4 \%$, respectivamente) 2 . No entanto, mundialmente, existe uma tendência de aumento do consumo de bebidas entre as mulheres 2 , as quais estão apresentando também maior mortalidade por câncer, doenças gastrointestinais e cardiovasculares 3. Diferenças socioeconômicas no consumo de álcool também são identificadas em diferentes países do mundo. Esse consumo tende a ser maior entre aqueles indivíduos com melhor nível socioeconômico, porém, os efeitos adversos do consumo abusivo de álcool tendem a ser maior entre os indivíduos com pior nível socioeconômico 2,3.

De acordo com a OMS, em 2010, 16\% da população mundial com 15 anos ou mais apresentaram, ao menos, um comportamento de consumo abusivo de álcool no período de um ano ${ }^{2}$. A OMS caracteriza o consumo abusivo de álcool, em ambos os sexos, pela ingestão de $60 \mathrm{~g}$ de álcool ou mais em uma única ocasião no período de 30 dias. Destaca-se que o consumo abusivo de álcool é um fator de risco para diferentes doenças crônicas, comportamentos violentos, acidentes de trânsito e transtornos mentais, acarretando assim prejuízos sociais, como altos custos para o sistema de saúde 2 .

Considerando que o consumo abusivo de álcool no Brasil é um importante problema de saúde pública, diferentes medidas para inibir este consumo têm sido adotadas. O aumento da tributação de bebidas alcoólicas, além de uma série de programas e medidas educacionais, foi adotado com o objetivo de diminuir o consumo abusivo de álcool e suas consequências adversas na sociedade brasileira. Soma-se a isso o monitoramento realizado pela pesquisa Vigilância de Fatores de Risco e Proteção para Doenças Crônicas por Inquérito Telefônico (VIGITEL). Esse monitoramento ocorre anualmente e inclui uma série de indicadores relacionados à saúde, entre eles o consumo abusivo de álcool. Apesar de esse monitoramento ser realizado anualmente, não foram localizados estudos que tenham avaliado a tendência do consumo abusivo de álcool no Brasil para a população adulta. Portanto, o objetivo do presente trabalho foi avaliar a tendência geral, por sexo, idade, escolaridade e por região para o consumo abusivo de álcool no Brasil entre 2006 e 2013.

\section{Metodologia}

\section{Local e desenho do estudo}

Este trabalho analisou dados do VIGITEL entre os anos de 2006 e 2013. O VIGITEL foi implantado pelo Ministério da Saúde no ano de 2006, e realiza o monitoramento anual da população adulta $(\geq 18$ anos) brasileira residente nas capitais dos 26 estados e, também, no Distrito Federal. Trata-se de um inquérito telefônico que inclui na amostra apenas as linhas de telefone fixo. Cerca de 54 mil indivíduos responderam ao inquérito a cada ano.

\section{Amostragem}

O processo amostral do VIGITEL foi planejado para incluir aproximadamente 2 mil entrevistas telefônicas por capital em cada ano de acompanhamento, tornando possível estimativas com erro amostral de cerca de dois pontos percentuais. Trata-se de amostragem probabilística, realizada em 
dois estágios: primeiro é realizado o sorteio sistemático de 5 mil linhas telefônicas para cada cidade, com a realização de 25 subamostras de 200 linhas cada. Após esse procedimento, é realizada aleatoriamente a escolha de um morador do domicílio selecionado com idade $\geq 18$ anos para responder ao questionário.

\section{Definição do desfecho}

O consumo abusivo de álcool foi avaliado pelo VIGITEL em todos os anos incluídos na presente análise utilizando o autorrelato dos entrevistados. O VIGITEL considera como consumo abusivo de álcool a ingestão de cinco ou mais doses (homens) ou quatro ou mais doses (mulheres) em uma única ocasião, pelo menos uma vez nos 30 dias anteriores à entrevista. Considerou-se para identificar o consumidor abusivo todos aqueles que responderam positivamente às seguintes questões, para os homens: "Nos últimos 30 dias, o Sr. chegou a consumir 5 ou mais doses de bebida alcoólica em uma única ocasião?"; para as mulheres, "Nos últimos 30 dias, a Sra. chegou a consumir 4 ou mais doses de bebida alcoólica em uma única ocasião?”. De acordo com o VIGITEL, uma dose de bebida alcoólica corresponde a uma lata de cerveja, uma taça de vinho ou uma dose de cachaça, uísque ou qualquer outra bebida alcoólica destilada. Para cada ano, foi calculada a proporção de adultos que consumiram bebida alcoólica de forma abusiva.

\section{Análise estatística}

Foram realizadas análises descritivas sobre o perfil da amostra e a proporção do consumo abusivo de álcool entre os anos de 2006 e 2013, de acordo com o sexo (masculino e feminino), idade em anos (categorizada em 18-29; 30-39; 40-49; 50-59; 60-69; $\geq 70$ ), escolaridade em anos completos (categorizada em $0-4 ; 5-8 ; 9-11 ; \geq 12$ ) e região do país (Norte, Nordeste, Centro-oeste, Sudeste e Sul).

Todas as análises foram realizadas no software Stata versão 12.1 (StataCorp LP, College Station, Estados Unidos) e utilizaram o comando svyset, que considera os pesos amostrais. Resumidamente, de acordo com os relatórios do VIGITEL, o peso final é calculado pelo método rake 4,5,6,7. Esse peso final é atribuído a cada indivíduo entrevistado no estudo para cada uma das 27 cidades e é o resultado de três fatores: (1) o inverso do número de linhas telefônicas no domicílio do entrevistado; (2) o número de adultos residentes no domicílio do entrevistado; e (3) o peso pós-estratificação, que visa a igualar a composição sociodemográfica (sexo, faixa etária e escolaridade) da amostra em relação à população adulta total da cidade. Informações detalhadas da composição desse fator de ponderação podem ser localizadas on-line no Departamento de Informática do SUS (DATASUS; http://tabnet.datasus.gov.br/ cgi/vigitel/vigteldescr.htm) e também na Biblioteca Virtual de Saúde (BVS; http://bvsms.saude.gov. br). Os testes de significância estatística para as tendências de consumo abusivo de álcool foram realizados utilizando-se o procedimento proposto por Prais-Winsten ${ }^{8}$. Esse procedimento é baseado na análise de regressão linear generalizada, sendo recomendado para análise de significância em estudos de tendência temporal, uma vez que o procedimento considera a autocorrelação serial, frequente em medidas de dados populacionais, que não é considerada, por exemplo, pelos métodos de regressão tradicionais (linear, logística e Poisson) 8. Todas as estimativas com nível de significância $<0,05$ indicam: aumento na tendência quando a mudança anual for positiva e redução na tendência quando a mudança anual for negativa. Quando não foi identificada uma diferença estatisticamente significativa, considerou-se que a tendência foi estacionária.

\section{Aspectos éticos}

De acordo com as informações dos relatórios do VIGITEL, por se tratar de entrevista por telefone, o consentimento livre e esclarecido foi substituído pelo consentimento verbal obtido por ocasião dos contatos telefônicos com os entrevistados. O VIGITEL foi aprovado pela Comissão Nacional de Ética em Pesquisa em Seres Humanos para cada ano do estudo. No entanto, nos relatórios oficiais só está disponível o número do parecer para o ano de 2013 (CONEP: parecer 355.590, de 26 de junho de 2013 - CAAE: 16202813.2.0000.0008). 


\section{Resultados}

O VIGITEL entrevistou um total de 425.865 brasileiros (53,9\% mulheres) no período em estudo. Entre 2006 e 2013, a proporção de indivíduos com idade entre 18 e 29 anos variou entre 31,8\% e $28,2 \%$, de pessoas com 9 a 11 anos de estudos variou entre 33,1\% e 36,8\%, e de residentes na Região Norte variou entre 9,2\% e 10\%, respectivamente (Tabela 1).

Foi observada uma tendência estacionária de consumo abusivo de álcool entre 2006 e 2013 para toda a amostra e ambos os sexos (Figura 1). Observou-se aumento no consumo abusivo de álcool entre as faixas etárias de 30 a 39 anos $(\mathrm{p}=0,05), 50$ a $59(\mathrm{p}=0,05)$ e 60 a 69 anos $(\mathrm{p}=0,01)$ e uma tendência estacionária para os demais grupos etários (Tabela 2). Verificou-se uma tendência estacionária de acordo com a escolaridade e a região de residência, exceto para a Região Sudeste, onde observou-se um aumento de 2,1\% no consumo abusivo de álcool no período. Destaca-se que o consumo abusivo de álcool foi maior na medida em que aumenta a escolaridade dos indivíduos, em todos os períodos em estudo. Para todos os anos pesquisados, as menores prevalências de consumo abusivo de álcool foram observadas na Região Sul e as maiores na Região Nordeste (Tabela 2).

Anualmente, um aumento de 0,7\% e 5,3\% foi identificado entre homens de 30 a 39 anos (p=0,05) e de 60 a $69(\mathrm{p}=0,02)$, respectivamente. Entre os homens com idade $\geq 70$ anos foi identificado um aumento de 10,2\% no consumo abusivo de álcool $(\mathrm{p}=0,04)$. Observou-se uma tendência estacionária no consumo abusivo de álcool para os homens de acordo com o nível de escolaridade e região do país (Tabela 2).

Houve um aumento de 3,8\% ao ano para as mulheres com idades entre 30 e 39 anos ( $\mathrm{p}=0,02)$, de $8,2 \%$ para aquelas com idades entre 50 e $59(\mathrm{p}=0,03)$, de $11,3 \%$ para as que tinham entre 60 e 69 ( $\mathrm{p}=0,01)$ e de $12,7 \%$ para aquelas com 70 anos ou mais $(\mathrm{p}=0,01)$. Verificou-se uma tendência estacionária de consumo abusivo de álcool para as mulheres em todos os grupos de escolaridade analisados. Foi observado um aumento de 3,9\% ao ano no consumo abusivo de álcool entre 2006 e 2013 para as mulheres da Região Sudeste ( $\mathrm{p}=0,03$ ) (Tabela 2).

\section{Tabela 1}

Descrição da amostra. Vigilância de Fatores de Risco e Proteção para Doenças Crônicas por Inquérito Telefônico (VIGITEL), Brasil, 2006-2013.

\begin{tabular}{|c|c|c|c|c|c|c|c|c|}
\hline \multirow[t]{2}{*}{ Variável } & \multicolumn{8}{|c|}{$\%$} \\
\hline & 2006 & 2007 & 2008 & 2009 & 2010 & 2011 & 2012 & 2013 \\
\hline \multicolumn{9}{|l|}{ Idade (anos) } \\
\hline $18-29$ & 31,8 & 31,5 & 31,5 & 30,2 & 30,0 & 29,6 & 28,6 & 28,2 \\
\hline $30-39$ & 22,2 & 21,9 & 21,4 & 21,8 & 21,8 & 21,7 & 22,3 & 22,0 \\
\hline $40-49$ & 18,8 & 18,8 & 18,6 & 18,9 & 18,5 & 18,9 & 18,6 & 18,7 \\
\hline $50-59$ & 13,2 & 13,5 & 14,1 & 14,3 & 14,4 & 14,4 & 14,9 & 14,9 \\
\hline $60-69$ & 7,9 & 8,2 & 8,3 & 8,4 & 8,8 & 8,9 & 8,8 & 9,0 \\
\hline$\geq 70$ & 6,0 & 6,2 & 6,2 & 6,3 & 6,4 & 6,5 & 6,8 & 7,1 \\
\hline \multicolumn{9}{|c|}{ Escolaridade (anos completos) } \\
\hline $0-4$ & 21,6 & 20,4 & 19,5 & 24,4 & 22,8 & 23,0 & 21,7 & 20,3 \\
\hline $5-8$ & 24,0 & 24,5 & 24,3 & 20,4 & 20,7 & 18,9 & 18,1 & 18,8 \\
\hline $9-11$ & 33,1 & 35,3 & 34,6 & 34,9 & 35,1 & 35,7 & 37,6 & 36,8 \\
\hline$\geq 12$ & 21,3 & 19,8 & 21,6 & 20,3 & 21,3 & 22,4 & 22,6 & 24,0 \\
\hline \multicolumn{9}{|l|}{ Região } \\
\hline Norte & 9,2 & 9,6 & 9,4 & 9,4 & 9,8 & 9,8 & 9,8 & 10,0 \\
\hline Nordeste & 24,1 & 25,0 & 25,4 & 25,5 & 25,0 & 25,1 & 26,0 & 25,1 \\
\hline Centro-oeste & 13,3 & 10,9 & 11,1 & 11,2 & 11,2 & 11,3 & 11,2 & 11,4 \\
\hline Sudeste & 45,0 & 45,9 & 45,6 & 45,4 & 45,8 & 45,6 & 44,9 & 45,4 \\
\hline Sul & 8,4 & 8,5 & 8,5 & 8,5 & 8,2 & 8,2 & 8,0 & 8,1 \\
\hline
\end{tabular}




\section{Figura 1}

Tendência do consumo abusivo de álcool para a população brasileira e de acordo com o sexo. Vigilância de Fatores de Risco e Proteção para Doenças Crônicas por Inquérito Telefônico (VIGITEL), Brasil, 2006-2013 ( $n=425.865)$.

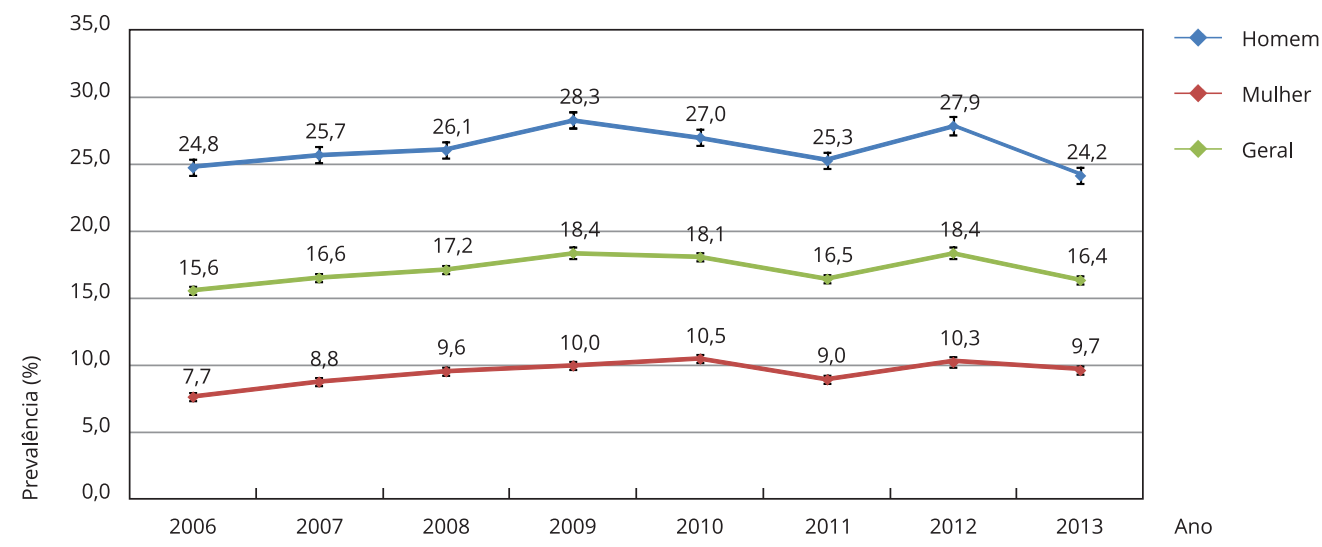

Nota: valor de p: geral =0,33; homens $=0,75 ;$ mulheres $=0,08$

\section{Tabela 2}

Prevalência e proporção da mudança anual no consumo abusivo de álcool para homens $(n=166.087)$ e mulheres $(n=259.778)$. Vigilância de Fatores de Risco e Proteção para Doenças Crônicas por Inquérito Telefônico (VIGITEL), Brasil, 2006-2013.

\begin{tabular}{|c|c|c|c|c|c|c|c|c|c|c|}
\hline \multirow[t]{2}{*}{ Variáveis } & \multicolumn{8}{|c|}{$\%$} & \multirow[t]{2}{*}{ Mudança anual (\%) } & \multirow[t]{2}{*}{ Valor de $p$ * } \\
\hline & 2006 & 2007 & 2008 & 2009 & 2010 & 2011 & 2012 & 2013 & & \\
\hline \multicolumn{11}{|l|}{ Toda a amostra } \\
\hline \multicolumn{11}{|l|}{ Idade (anos) } \\
\hline $18-29$ & 20,9 & 22,5 & 22,2 & 24,2 & 23,7 & 21,3 & 23,6 & 20,7 & 0,1 & 0,93 \\
\hline $30-39$ & 18,7 & 19,7 & 20,0 & 21,0 & 21,6 & 19,1 & 21,9 & 20,6 & 1,3 & 0,05 \\
\hline $40-49$ & 15,6 & 16,0 & 18,1 & 19,3 & 18,3 & 16,8 & 18,7 & 16,6 & 1,1 & 0,45 \\
\hline $50-59$ & 10,3 & 11,6 & 13,5 & 14,7 & 14,0 & 13,6 & 15,8 & 13,4 & 4,1 & 0,05 \\
\hline $60-69$ & 4,9 & 6,3 & 6,9 & 7,5 & 7,9 & 8,1 & 8,3 & 8,0 & 6,8 & 0,01 \\
\hline$\geq 70$ & 1,5 & 1,8 & 2,2 & 2,5 & 2,8 & 3,3 & 3,4 & 2,4 & 8,8 & 0,05 \\
\hline \multicolumn{11}{|c|}{ Escolaridade (anos completos) } \\
\hline $0-4$ & 10,1 & 9,1 & 10,7 & 11,7 & 11,4 & 10,7 & 12,6 & 9,8 & 2,4 & 0,06 \\
\hline $5-8$ & 16,7 & 18,2 & 17,6 & 18,7 & 18,0 & 17,5 & 19,3 & 17,1 & 0,5 & 0,14 \\
\hline $9-11$ & 17,0 & 18,6 & 19,2 & 19,8 & 19,7 & 17,7 & 19,3 & 17,5 & 0,2 & 0,88 \\
\hline$\geq 12$ & 17,8 & 18,9 & 19,5 & 23,9 & 22,7 & 19,7 & 21,9 & 19,6 & 1,6 & 0,36 \\
\hline \multicolumn{11}{|l|}{ Região } \\
\hline Norte & 17,2 & 17,3 & 19,7 & 18,4 & 18,3 & 15,9 & 16,5 & 14,5 & $-2,4$ & 0,11 \\
\hline Nordeste & 18,4 & 19,2 & 19,9 & 20,8 & 20,8 & 19,2 & 20,4 & 18,0 & 0,0 & 0,96 \\
\hline Centro-oeste & 15,4 & 17,0 & 17,3 & 18,6 & 18,7 & 15,1 & 19,3 & 17,1 & 1,1 & 0,32 \\
\hline Sudeste & 14,2 & 15,3 & 16,0 & 17,6 & 16,9 & 15,9 & 18,1 & 16,2 & 2,1 & 0,05 \\
\hline Sul & 13,3 & 14,5 & 12,8 & 15,4 & 15,3 & 14,1 & 15,2 & 13,4 & 0,9 & 0,28 \\
\hline
\end{tabular}

(continua) 
Tabela 2 (continuação)

\begin{tabular}{|c|c|c|c|c|c|c|c|c|c|c|}
\hline \multirow[t]{2}{*}{ Variáveis } & \multicolumn{8}{|c|}{$\%$} & \multirow[t]{2}{*}{ Mudança anual (\%) } & \multirow[t]{2}{*}{ Valor de $p$ * } \\
\hline & 2006 & 2007 & 2008 & 2009 & 2010 & 2011 & 2012 & 2013 & & \\
\hline \multicolumn{11}{|l|}{ Homens } \\
\hline \multicolumn{11}{|l|}{ Idade (anos) } \\
\hline $18-29$ & 30,7 & 32,0 & 30,6 & 34,4 & 31,9 & 30,4 & 32,0 & 26,8 & $-1,0$ & 0,23 \\
\hline $30-39$ & 29,5 & 30,7 & 30,4 & 31,7 & 32,3 & 29,5 & 33,2 & 30,4 & 0,7 & 0,05 \\
\hline $40-49$ & 24,1 & 23,5 & 27,2 & 29,1 & 27,9 & 25,0 & 27,8 & 24,8 & 0,8 & 0,56 \\
\hline $50-59$ & 18,1 & 21,6 & 22,6 & 24,0 & 21,5 & 21,2 & 26,3 & 21,5 & 2,2 & 0,10 \\
\hline $60-69$ & 10,0 & 11,1 & 12,3 & 14,6 & 13,9 & 14,3 & 14,5 & 14,4 & 5,3 & 0,02 \\
\hline$\geq 70$ & 2,7 & 3,1 & 3,9 & 5,0 & 5,4 & 5,9 & 6,8 & 4,4 & 10,2 & 0,04 \\
\hline \multicolumn{11}{|c|}{ Escolaridade (anos completos) } \\
\hline $0-4$ & 18,0 & 15,6 & 19,0 & 19,6 & 18,9 & 18,6 & 21,5 & 15,9 & 2,0 & 0,08 \\
\hline $5-8$ & 25,8 & 28,2 & 26,5 & 28,2 & 26,8 & 26,5 & 28,9 & 25,8 & 0,1 & 0,65 \\
\hline $9-11$ & 26,1 & 28,2 & 28,5 & 30,4 & 28,9 & 25,9 & 28,7 & 25,2 & $-0,6$ & 0,55 \\
\hline$\geq 12$ & 28,3 & 28,1 & 27,8 & 35,2 & 32,9 & 30,2 & 32,0 & 28,4 & 1,0 & 0,52 \\
\hline \multicolumn{11}{|l|}{ Região } \\
\hline Norte & 27,6 & 28,1 & 31,1 & 29,1 & 29,5 & 24,7 & 25,8 & 22,8 & $-2,86$ & 0,07 \\
\hline Nordeste & 29,8 & 30,1 & 31,3 & 33,0 & 31,7 & 29,3 & 31,9 & 27,5 & $-0,51$ & 0,54 \\
\hline Centro-oeste & 23,7 & 26,3 & 26,6 & 25,2 & 27,2 & 22,3 & 28,0 & 24,9 & 0,07 & 0,91 \\
\hline Sudeste & 22,4 & 23,2 & 23,4 & 27,1 & 24,5 & 24,7 & 27,0 & 23,1 & 1,50 & 0,09 \\
\hline Sul & 22,1 & 22,6 & 19,4 & 24,1 & 23,4 & 21,6 & 23,4 & 21,2 & 0,46 & 0,56 \\
\hline \multicolumn{11}{|l|}{ Mulheres } \\
\hline \multicolumn{11}{|l|}{ Idade (anos) } \\
\hline $18-29$ & 11,2 & 13,1 & 13,8 & 14,1 & 15,4 & 12,0 & 14,8 & 14,3 & 2,2 & 0,13 \\
\hline $30-39$ & 9,0 & 9,6 & 10,6 & 11,6 & 12,3 & 10,3 & 12,3 & 11,9 & 3,8 & 0,02 \\
\hline $40-49$ & 8,4 & 9,7 & 10,8 & 11,2 & 10,3 & 9,9 & 10,8 & 9,7 & 1,6 & 0,37 \\
\hline $50-59$ & 4,4 & 4,9 & 6,5 & 7,4 & 8,1 & 7,5 & 7,8 & 7,6 & 8,2 & 0,03 \\
\hline $60-69$ & 1,4 & 2,5 & 2,8 & 2,9 & 3,4 & 3,7 & 3,9 & 3,2 & 11,3 & 0,01 \\
\hline$\geq 70$ & 0,6 & 0,8 & 1,1 & 0,7 & 1,3 & 1,6 & 1,5 & 1,2 & 12,7 & 0,01 \\
\hline \multicolumn{11}{|c|}{ Escolaridade (anos completos) } \\
\hline $0-4$ & 3,9 & 4,0 & 4,4 & 5,2 & 5,0 & 4,2 & 5,4 & 4,7 & 3,3 & 0,05 \\
\hline $5-8$ & 8,1 & 8,8 & 9,1 & 9,8 & 10,0 & 9,1 & 9,7 & 8,9 & 1,4 & 0,26 \\
\hline $9-11$ & 9,2 & 10,3 & 11,3 & 10,7 & 11,9 & 10,5 & 11,1 & 10,7 & 1,5 & 0,19 \\
\hline$\geq 12$ & 9,0 & 11,3 & 12,4 & 14,8 & 14,3 & 11,4 & 14,1 & 12,9 & 4,2 & 0,13 \\
\hline \multicolumn{11}{|l|}{ Região } \\
\hline Norte & 7,7 & 7,4 & 9,2 & 8,6 & 8,1 & 7,9 & 8,0 & 7,0 & $-1,0$ & 0,52 \\
\hline Nordeste & 9,0 & 10,2 & 10,5 & 10,7 & 11,9 & 10,9 & 10,9 & 10,1 & 1,6 & 0,27 \\
\hline Centro-oeste & 8,1 & 8,7 & 9,1 & 12,8 & 11,2 & 8,6 & 11,5 & 10,2 & 3,3 & 0,20 \\
\hline Sudeste & 7,2 & 8,5 & 9,7 & 9,6 & 10,4 & 8,5 & 10,5 & 10,4 & 3,9 & 0,03 \\
\hline Sul & 5,8 & 7,5 & 7,1 & 8,0 & 8,3 & 7,7 & 8,2 & 6,8 & 2,2 & 0,20 \\
\hline
\end{tabular}

* Teste de Wald de tendência linear obtido pela regressão de Prais-Winsten.

\section{Discussão}

Foi observada alta prevalência e uma tendência estacionária de consumo abusivo de álcool no Brasil entre 2006 e 2013 para ambos os sexos. No entanto, a prevalência foi maior entre os homens do que entre as mulheres em todo o período. Foi identificado um padrão de menor frequência do consumo abusivo na medida em que aumentava a idade, porém, foi observada uma tendência de aumento deste comportamento entre os idosos de ambos os sexos e entre os homens de 30 a 39 anos. Observou-se uma tendência de aumento do consumo abusivo de álcool para as mulheres da Região Sudeste e 
uma tendência estacionária, para ambos os sexos, nas outras regiões do país. Tendência estacionária também foi verificada entre os diferentes grupos de escolaridade, embora a prevalência de consumo abusivo de álcool seja maior à proporção que aumenta a escolaridade.

A prevalência do consumo abusivo de álcool no Brasil é similar àquelas reportadas em diferentes países do mundo, indicando alta prevalência, padrão de ocorrência maior entre os homens, entre os indivíduos mais jovens e entre aqueles com melhor nível socioeconômico e maior escolaridade 2. Nos Estados Unidos, Dawson et al. 9 identificaram um aumento no consumo abusivo de álcool comparando os anos de 2001/2002 com 2012/2013. Esse aumento foi maior entre as mulheres, entre aqueles indivíduos com 25 a 44 anos e naqueles com idade $\geq 65$ anos. Na Austrália, Livingston et al. 10 analisaram informações de cinco inquéritos nacionais com amostragem probabilística (National Drug Strategy Household Survey) realizados em 2001 ( $\mathrm{n}=26.744 ; \geq 14$ anos), 2004 ( $\mathrm{n}=29.455 ; \geq 12$ anos), 2007 ( $\mathrm{n}=23.356 ; \geq 12$ anos), 2010 ( $\mathrm{n}=26.648 ; \geq 14$ anos) e 2013 ( $\mathrm{n}=23.855 ; \geq 12$ anos). Esses autores reportaram um aumento no volume de álcool consumido anualmente e identificaram um declínio entre os anos de 2007 e 2013. Aproximadamente 30\% dessa redução no consumo ocorreram entre os indivíduos mais jovens ( $\leq 30$ anos). Esses autores identificaram um aumento de 5,8\% nos indivíduos com idades entre 60 e 69 anos no período estudado 10. Não foram identificadas na literatura explicações para o aumento do consumo abusivo de álcool entre os indivíduos com maior idade. Apenas um estudo, realizado nos Estados Unidos, identificou que os efeitos de problemas econômicos (como perder o emprego/casa/aposentadoria e a redução do salário, por exemplo) estão associados ao maior consumo de álcool em indivíduos com 50 anos ou mais ${ }^{11}$. Na Dinamarca, Bjork et al. 12 avaliaram a tendência (nos anos de 1987, 1994, 2000, 2003 e 2005) de consumo de álcool na última semana, em indivíduos entre 50 e 74 anos de idade $(\mathrm{n}=15.144)$. Foi identificada uma tendência de aumento entre os mais jovens, em ambos os sexos, no período estudado, porém o padrão de consumo foi menor na medida em que aumentava a idade 12. Na Espanha, Galán et al. 13 analisaram dados de inquérito telefônico nacional realizado entre 1995 e 2003 com indivíduos de 18 a 64 anos $(\mathrm{n}=18.049)$. O consumo abusivo de álcool foi definido como a ingestão de $50 \mathrm{~mL}$ para homens e $30 \mathrm{~mL}$ para mulheres, na última semana. Houve uma diminuição no consumo abusivo de álcool de 6,1\% no período, para os homens, maior entre aqueles de 18 a 44 anos e com nível de escolaridade média-alta 13. Na Alemanha, Völzke et al. 14 avaliaram a tendência de consumo abusivo de álcool entre 1997-2001 e 2008-2012 $(\mathrm{n}=8.728)$. Os autores identificaram um declínio na média de uso de álcool pela população (de 5,57g/ dia para 3,22g/dia) no período 14 . Na Suécia, Kraus et al. 15 analisaram a tendência de consumo de álcool (últimos 30 dias e volume de álcool consumido) em indivíduos com idades de 16 a 80 anos ( $\mathrm{n}=77.598)$ entre os anos de 1979 e 2011. Foi observado um aumento no volume de álcool consumido entre 1979 e 2005, e uma estabilização no consumo entre 2005 e 2011, para ambos os sexos. O consumo foi maior entre indivíduos de 40 a 50 anos e entre os homens, em todos os períodos ${ }^{15}$. Na Inglaterra, Meng et al. 16 avaliaram a tendência de consumo de álcool em indivíduos com idade $\geq 16$ $\operatorname{anos}(n=200.144)$ entre 1984 e 2009. O consumo foi maior entre jovens (18-24 anos) e foi observada uma tendência de aumento entre as mulheres ${ }^{16}$. No Brasil, resultados da Pesquisa Nacional de Saúde (PNS) sobre consumo abusivo de álcool indicaram um padrão de ocorrência deste comportamento similar ao observado no presente estudo 17 , isto é, maior entre os homens e entre os indivíduos mais jovens. Na Holanda, Geels et al. 18 analisaram dados de 16.587 indivíduos com idades entre 18 e 97 anos, com registros no sistema de saúde entre os anos de 2009-2012 e identificaram um aumento do consumo abusivo de álcool entre indivíduos com idade $\geq 65$ anos 18 . O crescimento do consumo de álcool entre essa faixa etária foi reportado como resultado do aumento na expectativa de vida e aumento da renda da população 18,19 .

As diferenças entre os padrões de consumo abusivo de álcool dentre os sexos, que ocorre especialmente com adolescentes e indivíduos mais jovens 20 , envolvem uma série de fatores complexos, incluindo características biológicas e sociais 21 . A hipótese mais aceita para explicar a crescente tendência do consumo abusivo de álcool entre as mulheres está relacionada à maior participação nas atividades tradicionalmente relegadas aos homens, especialmente em relação ao mercado de trabalho e educação 20,21. Segundo essa hipótese, essas características são acompanhadas de um crescimento da autonomia das mulheres, possibilitando e encorajando o aumento do consumo de álcool e, consequentemente, o aumento da quantidade e frequência do consumo. Porém, a relação causal entre a atuação no mercado de trabalho e maior consumo de álcool não está explicada na literatura 21. Outra 
hipótese sugere maior diferença no padrão do consumo de álcool entre os sexos de acordo com o nível de desenvolvimento econômico do ambiente social no qual as mulheres se encontram ${ }^{20}$. Assim como para a primeira hipótese, explicações empiricamente e teoricamente fundamentadas ainda são insuficientes. A literatura nessa área recomenda que estudos voltados para entender essas diferenças entre os sexos sejam conduzidos em contextos socioculturais diversos e que, preferencialmente, sejam realizados durante longos períodos de forma a evitar a simplificação das conclusões relacionadas a essas diferenças 21 .

Os prejuízos decorrentes do consumo abusivo de álcool geram alto custo para os sistemas de saúde, especialmente em um país com uma dimensão populacional como o Brasil. Em 2015, de acordo com a Coordenação Geral de Saúde Mental, Álcool e Outras Drogas do Ministério da Saúde, as mortes relacionadas ao consumo abusivo de álcool e dependência de álcool correspondem a 93,5\% do total de óbitos por causa básica atribuída ao uso de substâncias psicoativas 22 . Portanto, estratégias para a diminuição do consumo abusivo de álcool são essenciais para minimizar impactos na saúde no âmbito individual e coletivo, incluindo a redução da sobrecarga no sistema de saúde devido a agravos relacionados ao consumo abusivo de álcool. De acordo com a OMS, o aumento dos preços das bebidas alcoólicas é uma medida custo-efetiva para a redução do consumo abusivo de álcool 23. Existem evidências de que o controle rígido sobre o acesso a bebidas alcoólicas (incluindo a restrição do horário para a venda e controle do governo sobre a venda a varejo) e maior regulação e restrição dos pontos de venda são medidas favoráveis ao enfrentamento do consumo abusivo de álcool 23. Algumas estratégias de saúde pública foram definidas pelo Governo Federal nos últimos anos. No ano de 2003, foi publicada a Política Nacional de Promoção da Saúde, que prevê, entre outras iniciativas, a redução da morbimortalidade em decorrência do uso abusivo de álcool e outras drogas. Em 2007, foi publicado o decreto que aprovou a Política Nacional sobre Álcool (PNA), que "dispõe sobre as medidas para redução do uso indevido de álcool e sua associação com a violência e criminalidade” 24. Em 2009, foi instituído o Plano Emergencial de Ampliação do Acesso ao Tratamento e Prevenção em Álcool e outras Drogas no Sistema Único de Saúde 25. Evidências sugerem que houve uma redução da associação entre consumo de álcool e condução de veículos motorizados no Brasil entre 2007-2008 e 2012-2013 26, possivelmente resultado das políticas de combate ao uso de álcool e a condução de veículos motorizados, bem como do aumento da fiscalização deste comportamento.

As estratégias relacionadas à redução do uso de álcool e prevenção dos danos à saúde e à vida, propostas pela PNA, incluem o diagnóstico do consumo no Brasil, o incentivo à regulamentação, o monitoramento e a fiscalização da propaganda e publicidade de bebidas alcoólicas a segmentos populacionais vulneráveis, o tratamento e reinserção de usuários e dependentes de álcool, a realização de campanhas de informação, a sensibilização e mobilização da opinião pública quanto às consequências do uso indevido e do abuso de bebidas alcoólicas, a redução da demanda de álcool por populações vulneráveis, medidas para a fiscalização e prevenção de condução de veículos motorizados sob o efeito de álcool, bem como o estabelecimento de parceria com os municípios para a recomendação de ações municipais 24 . No entanto, a proporção de consumo abusivo de álcool foi maior entre indivíduos com maior escolaridade e que, provavelmente, apresentam maior nível socioeconômico. O monitoramento e a sistematização de informações sobre as estratégias propostas pela PNA são extremamente necessários e úteis para auxiliar a identificação de ações efetivas, o planejamento de novas ações e a descontinuidade de estratégias não efetivas. Entretanto, essas informações ainda não estão disponíveis no momento atual, tratando-se de um desafio para o enfrentamento do consumo abusivo de álcool e seus prejuízos à saúde pública e agravos à saúde individual. Outras estratégias da PNA para diminuir o consumo abusivo de álcool pela população devem ser priorizadas, com a implantação e avaliação de estratégias propostas pela OMS, que incluem o controle rígido sobre o acesso às bebidas alcoólicas e maior regulação e restrição dos pontos de venda, assim como estratégias de prevenção.

Recentemente, o Ministério da Saúde brasileiro iniciou ações preventivas e de promoção da saúde entre crianças, jovens e famílias 22. Três programas de prevenção do uso e abuso de álcool, tabaco, crack e outras drogas vêm sendo realizados e avaliados em escolas brasileiras 22. O Jogo Elos, implantado em sala de aula pelos professores e com foco em crianças de 6 a 10 anos, estimula atitudes positivas e colaborativas, favorecendo o desenvolvimento de habilidades sociais, como o autocontrole, empatia, assertividade, entre outras 22. O programa Tamo Junto, voltado para adolescentes de 11 a 14 anos, combina atividades realizadas por professores com atividades em conjunto com os pais, e tem como 
objetivo o desenvolvimento de habilidades de vida e pensamento crítico frente às crenças normativas e informações a respeito de estilos de vida dos adolescentes 22 . O programa Fortalecendo Famílias é realizado por profissionais que trabalham nos Centros de Referência em Assistência Social (CRAS) e consiste em sete encontros semanais com as famílias, com o objetivo de promover a reflexão sobre valores e rotinas familiares, além de fortalecer os vínculos entre pais e filhos, habilidade de expressão de sentimentos e o estabelecimento de regras 22. Segundo o Ministério da Saúde, a avaliação desses programas está em andamento e ainda não estão publicados os resultados dessas intervenções. Se custo-efetivas, contribuirão para a redução do uso abusivo de álcool e outras drogas em longo prazo 22.

Entre as vantagens deste trabalho, destacam-se o seu caráter essencial de monitoramento das condições de saúde da população das capitais brasileiras, a análise da tendência temporal desse comportamento e a possibilidade de contribuição ao planejamento de políticas e programas de saúde voltados ao enfrentamento do consumo abusivo de álcool. A principal limitação é decorrente do fato de a avaliação do consumo abusivo de álcool ser feita por autorrelato direto, sem mensuração da quantidade e frequência da utilização de álcool. Devido ao desenho do estudo, não é possível comparar se o consumo abusivo de álcool é diferente entre as zonas urbana e rural, bem como entre as capitais e as cidades do interior, o que poderia ser útil ao planejamento estratégico.

\section{Conclusões}

A prevalência do consumo abusivo de álcool nas capitais brasileiras é alta, principalmente entre os homens, entre aqueles com maior escolaridade e os mais jovens, e apresenta uma tendência estacionária, tanto para os homens como para as mulheres. As políticas nacionais podem ter contribuído para que o consumo abusivo de álcool tenha se mantido estável, no entanto, a sistematização das informações referentes às estratégias de enfrentamento ao consumo abusivo de álcool e a avaliação destas estratégias são necessárias para o planejamento de ações no âmbito da saúde pública. Estratégias de intervenção em saúde e prevenção ao consumo abusivo de álcool devem ser desenvolvidas e implantadas em curto prazo, de forma a minimizar os impactos que o consumo abusivo de álcool gera ao sistema de saúde e à sociedade brasileira.

\section{Colaboradores}

T. N. Munhoz participou da concepção do estudo, análise e interpretação dos dados, interpretação dos resultados e escrita de todas as versões do manuscrito. I. S. Santos, B. P. Nunes, C. L. Mola, I. C. M. Silva e A. Matijasevich participaram da concepção do estudo, interpretação dos dados, colaboração com a interpretação dos resultados e escrita de todas as versões do manuscrito. Todos os autores revisaram criticamente e aprovaram a versão final.

\section{Referências}

1. Nelson JP, McNall AD. Alcohol prices, taxes, and alcohol-related harms: a critical review of natural experiments in alcohol policy for nine countries. Health Policy 2016; 120:264-72.

2. World Health Organization. Global status report on alcohol and health 2014. Geneva: World Health Organization; 2014.

3. Rehm J, Baliunas D, Borges GL, Graham K, Irving $H$, Kehoe $T$, et al. The relation between different dimensions of alcohol consumption and burden of disease: an overview. Addiction 2010; 105:817-43.

4. Battaglia MP, Frankel MR, Link MW. Improving standard poststratification techniques for random-digit-dialing telephone surveys. Surv Res Methods 2008; 2:11-9. 
5. Bernal RTI. Inquéritos por telefone: inferências válidas em regiões com baixa taxa de cobertura de linhas residenciais [Tese de Doutorado]. São Paulo: Universidade de São Paulo; 2011.

6. Izrael D, Hoaglin DC, Battaglia MP. A SAS macro for balancing a weighted sample. In: Proceedings of the Twenty-Fifth Annual SAS Users Group International Conference. Cary: SAS Institute; 2000. p. 1350-5.

7. Izrael D, Hoaglin DC, Battaglia MP. To rake or not to rake is not the question anymore with the enhanced raking macro. In: Proceedings of the Twenty-Ninth Annual SAS Users Group International Conference; Cary: SAS Institute; 2004.

8. Antunes JLF, Cardoso MRA. Uso da análise de séries temporais em estudos epidemiológicos. Epidemiol Serv Saúde 2015; 24:565-76.

9. Dawson DA, Goldstein RB, Saha TD, Grant BF. Changes in alcohol consumption: United States, 2001-2002 to 2012-2013. Drug Alcohol Depend 2015; 148:56-61.

10. Livingston M, Dietze P. National survey data can be used to measure trends in population alcohol consumption in Australia. Aust N Z J Public Health 2016; 40:233-5.

11. Mulia N, Zemore SE, Murphy R, Liu H, Catalano R. Economic loss and alcohol consumption and problems during the 2008 to 2009 U.S. recession. Alcohol Clin Exp Res 2014; 38:1026-34.

12. Bjork C, Thygesen LC, Vinther-Larsen M, Gronbaek MN. Time trends in heavy drinking among middle-aged and older adults in Denmark. Alcohol Clin Exp Res 2008; 32:120-7.

13. Galán I, Rodríguez-Artalejo F, Tobías A, Gandarillas A, Zorrilla B. Vigilancia de los factores de riesgo de las enfermedades no transmisibles mediante encuesta telefónica: resultados de la Comunidad de Madrid en el período 1995 2003. Gac Sanit 2005; 19:193-205.

14. Volzke H, Ittermann T, Schmidt CO, Baumeister SE, Schipf S, Alte D, et al. Prevalence trends in lifestyle-related risk factors. Dtsch Arztebl Int 2015; 112:185-92.

15. Kraus L, Tinghog ME, Lindell A, Pabst A, Piontek D, Room R. Age, period and cohort effects on time trends in alcohol consumption in the Swedish adult population 1979-2011. Alcohol Alcohol 2015; 50:319-27.
16. Meng Y, Holmes J, Hill-McManus D, Brennan A, Meier PS. Trend analysis and modelling of gender-specific age, period and birth cohort effects on alcohol abstention and consumption level for drinkers in Great Britain using the General Lifestyle Survey 1984-2009. Addiction 2014; 109:206-15.

17. Garcia LP, Freitas LRS. Consumo abusivo de álcool no Brasil: resultados da Pesquisa $\mathrm{Na}$ cional de Saúde 2013. Epidemiol Serv Saúde 2015; 24:227-37.

18. Geels LM, Vink JM, van Beek JH, Bartels M, Willemsen G, Boomsma DI. Increases in alcohol consumption in women and elderly groups: evidence from an epidemiological study. BMC Public Health 2013; 13:207.

19. Blazer DG, Wu LT. The epidemiology of alcohol use disorders and subthreshold dependence in a middle-aged and elderly community sample. Am J Geriatr Psychiatry 2011; 19:685-94

20. Wilsnack RW, Vogeltanz ND, Wilsnack SC, Harris TR, Ahlstrom S, Bondy S, et al. Gender differences in alcohol consumption and adverse drinking consequences: cross-cultural patterns. Addiction 2000; 95:251-65.

21. World Health Organization. Alcohol, gender and drinking problems in low and middle income countries. Geneva: World Health Organization; 2005.

22. Ministério da Saúde. Saúde Mental em Dados 2015; 10(12).

23. World Health Organization. What are the most effective and cost-effective interventions in alcohol control? http://www.euro.who.int/ document/e82969.pdf (acessado em 18/Mai/ 2016).

24. Brasil. Decreto no 6.117 , de 22 de maio de 2007. Diário Oficial da União 2007; 23 mai.

25. Ministério da Saúde. Portaria no 1.190 , de 4 de junho de 2009. Diário Oficial da União 2009; 5 jun.

26. Malta DC, Berna RT, Silva MM, Claro RM, Silva Junior JB, Reis AA. Consumption of alcoholic beverages, driving vehicles, a balance of dry law, Brazil 2007-2013. Rev Saúde Pública 2014; 48:692-96. 


\section{Abstract}

The aim of this study was to evaluate the trend in alcohol abuse in Brazil from 2006 to 2013 according to demographic, socioeconomic, and regional characteristics. This was an analysis of cross-sectional studies (VIGITEL, the Risk and Protective Factors Surveillance for Chronic Non-Communicable Diseases through Telephone Interview) that evaluated the Brazilian adult population ( $\geq 18$ years) in the country's state capitals. Sampling was two-stage probabilistic (telephone line and adult resident). Alcohol abuse was defined as $\geq 5$ drinks for men and $\geq 4$ drinks for women on a single occasion, at least once in the 30 days prior to the interview. Trend analysis was obtained using Prais-Winsten regression. Prevalence of alcohol abuse was $15.6 \%$ in 2006 and $16.4 \%$ in 2013, with a stationary trend in the entire sample $(p=0.334)$ and in both sexes. There was an upward trend in the elderly and in the 30-39-year age bracket in both sexes and in women in the Southeast Region of the country. A stationary trend was observed in different groups according to schooling. No downward trends were observed during the period analyzed.

Alcohol Drinking; Binge Drinking; Dangerous Behavior

\section{Resumen}

Se tuvo como objetivo evaluar la tendencia del consumo abusivo de alcohol en Brasil entre 2006 y 2013, según las características demográficas, socioeconómicas y regionales. Se trata de un análisis de estudios transversales (VIGITEL -Vigilancia de Factores de Riesgo y Protección para Enfermedades Crónicas por Encuesta Telefónica) que evaluaron a la población adulta ( $\geq 18$ años) brasileña, residente en las capitales de diferentes estados del país. La muestra fue probabilistica, realizada en dos fases (línea telefónica $y$ residente adulto). Se consideró consumo abusivo de alcohol la ingestión de $\geq 5$ (hombre) $o \geq 4$ (mujer) dosis de alcohol en una única ocasión, al menos una vez durante los últimos 30 días anteriores a la entrevista. El análisis de tendencia se obtuvo usando la regresión de Prais-Winsten. La prevalencia de consumo abusivo de alcohol fue de un $15,6 \%$ en 2006 y un $16,4 \%$ en 2013, con una tendencia estacionaria para toda la muestra $(p=$ $0,334)$ y para ambos sexos. La tendencia fue creciente entre los ancianos y aquellos con 30-39 años en ambos sexos y para las mujeres de la región sudeste. La tendencia estacionaria se observó entre los diferentes grupos de escolaridad. No se observaron tendencias decrecientes durante el periodo evaluado.

Consumo de Bebidas Alcohólicas; Borrachera; Conducta Peligrosa
Recebido em 16/Jun/2016

Versão final reapresentada em 14/Set/2016

Aprovado em 22/Set/2016 